\title{
Emergency surgery after percutaneous transmitral commissurotomy: Operative versus echocardiographic findings, mechanisms of complications, and outcomes
}

Praveen Kerala Varma, Mch, ${ }^{\text {a }}$ Sanjay Theodore, MS, ${ }^{\text {a }}$ Praveen Kumar Neema, MD, ${ }^{\mathrm{b}}$ Padmakumar Ramachandran, $\mathrm{DM}^{\mathrm{c}}$
Harikrishnan Sivadasanpillai, DM, ${ }^{\mathrm{c}}$ Krishna Kumar Nair, DM, ${ }^{\mathrm{c}}$ and Kurur Sankaran Neelakandhan, $\mathrm{Mch}^{\mathrm{a}}$

From the Divisions of Cardio-Vascular and Thoracic Surgery, ${ }^{\mathrm{a}}$ Anesthesiology, ${ }^{\mathrm{b}}$ and Cardiology, ${ }^{\mathrm{c}}$ Sree Chitra Tirunal Institute for Medical Sciences and Technology, Trivandrum, Kerala, India.

Received for publication Sept 13, 2004; revisions received March 29, 2005; accepted for publication April 7, 2005.

Address for reprints: Praveen Kerala Varma, Mch, Division of Cardio-Vascular and Thoracic Surgery, Sree Chitra Tirunal Institute for Medical Sciences and Technology, Trivandrum, Kerala, India-695 011 (E-mail: pkvarma@sctimst.ker.nic.in, varmapk@ gmail.com)

J Thorac Cardiovasc Surg 2005;130:772-6

$0022-5223 / \$ 30.00$

Copyright (C) 2005 by The American Association for Thoracic Surgery

doi:10.1016/j.jtcvs.2005.04.021
Objective: This study was undertaken to determine the clinical profile of patients undergoing emergency surgery after balloon mitral valvotomy, to note operative findings and compare them with those of transthoracic echocardiography, to describe the mechanisms of complications, and to describe outcomes.

Methods: A retrospective study was undertaken of patients requiring emergency surgery after percutaneous mitral valvotomy with an Inoue balloon from January 1990 to December 2003. The data analyzed included demographic variables, causes and clinical presentations of complications, and outcome. In 14 consecutive cases of mitral regurgitation, an observational study comparing the operative findings with echocardiography was also undertaken.

Results: In 1388 cases of valvotomy, complications necessitating urgent surgery occurred in 31 cases (2.2\%). Acute mitral regurgitation occurred in 23 cases (74.2 $\%$ ), and cardiac tamponade occurred in 8 cases (25.8\%). Mitral regurgitation was due to leaflet tearing in all cases: anterior leaflet in 20 cases and posterior leaflet in 3 cases. Hypotension, orthopnea, and pulmonary edema were the clinical presentation for mitral regurgitation. Transthoracic echocardiography underestimated the severity of mitral valve pathology. Bilateral severe commissural fusion and pliable leaflet with paracommissural calcium was seen in anterior leaflet tearing. Cardiac tamponade with hemodynamic compromise occurred as a result of left atrial perforation in 6 cases, right atrial perforation in 1 case, and left ventricular perforation in 1 case. High septal puncture led to atrial perforation. Operative mortality was $9.6 \%$, and low cardiac output developed in $29 \%$.

Conclusion: Acute mitral regurgitation and cardiac tamponade were the causes of emergency surgery after balloon valvotomy. Transthoracic echocardiography underestimated the severity of valve pathology.

I noue and colleagues ${ }^{1}$ in 1984 described percutaneous transmitral commissurotomy (PTMC) as an effective alternative to closed mitral valvuloplasty (CMV) for mitral stenosis (MS). Reported complications of PTMC include mitral regurgitation (MR), cardiac tamponade (CT), complete heart block, thromboembolism, and left-to-right shunt across the septal puncture. ${ }^{2}$ Complications necessitating urgent surgery are rare ${ }^{3}$ and include acute, severe MR after disruption of the mitral valve apparatus ${ }^{3}$ and CT caused by cardiac chamber perforation. ${ }^{3}$

Although PTMC has been widely applied clinically, and potential complications have been defined, only few series have highlighted the clinical profile, surgical findings, complications of surgery, and outcomes of patients who undergo emergency surgery after PTMC. We present our experience with emergency surgery after PTMC complications, with a special emphasis on preoperative clinical presentation, 
TABLE 1. Patient demographics $(\mathbf{n}=\mathbf{3 1})$

\begin{tabular}{lc}
\hline Variable & Value \\
\hline Age (y) & \\
Mean \pm SD & $35.6 \pm 10.9$ \\
$\quad$ Range & $10-50$ \\
Body surface area $\left(\mathrm{m}^{2}\right)$ & \\
$\quad$ Mean \pm SD & $1.45 \pm 0.16$ \\
$\quad$ Range & $1.2-1.7$ \\
Height (cm) & \\
$\quad$ Mean \pm SD & $154.5 \pm 10.98$ \\
Range & $132-176$ \\
Sex (No.) & \\
Male & $9 / 31(29 \%)$ \\
Female & $22 / 31(71 \%)$ \\
Rhythm (No.) & \\
Atrial fibrillation & $17 / 31(54.8 \%)$ \\
Sinus rhythm & $14 / 31(43.2 \%)$ \\
New York Heart Association functional class (No.) & \\
I & 0 \\
II & $9 / 31(29 \%)$ \\
III & $20 / 31(64.5 \%)$ \\
IV & $2 / 31(6 \%)$ \\
Previous CMV (No.) & $3 / 31(9.6 \%)$ \\
\end{tabular}

CMV, Closed mitral valvuloplasty.

operative findings in comparison with transthoracic echocardiography (TTE), complications of the surgery, and outcomes. We also propose possible mechanisms of the complications according to the operative findings.

\section{Patients and Methods}

Between January 1990 and December 2003, a total of 1388 PTMCs were performed with Inoue balloons (Toray Medical, Tokyo, Japan). Involvement of the aortic valve, presence of more than Sellers grade $2 \mathrm{MR}$, severe commissural calcification of the valve, and left atrial clot excluded patients from PTMC.

\section{Collection of Data}

Retrospective analysis of patients requiring emergency surgery after PTMC was undertaken from case records. The data analyzed

TABLE 2. Clinical profiles of patients with complications

\begin{tabular}{lcc}
\hline & No. & $\%$ \\
\hline Total & 31 & \\
Severe MR & $23 / 31$ & 74.2 \\
Pulmonary edema & $5 / 23$ & 21.7 \\
Orthopnea & $6 / 23$ & 26.1 \\
Hypotension & $12 / 23$ & 52.2 \\
CT & $8 / 31$ & 25.8 \\
Cardiac arrest & $3 / 8$ & 37.5 \\
Hypotension & $8 / 8$ & 100 \\
Dyspnea & $8 / 8$ & 100 \\
\hline
\end{tabular}

$M R$, Mitral regurgitation; $C T$, cardiac tamponade.

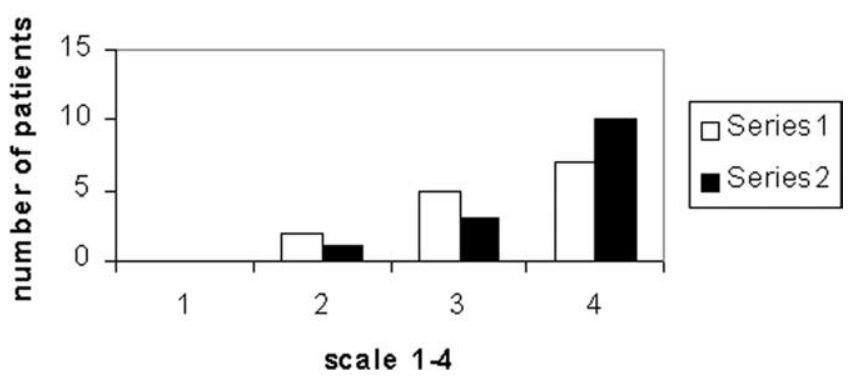

Figure 1. Comparison between TTE findings and operative findings with respect to subvalvular pathology in 14 consecutive cases of MR. Series 1 (white bars) represents TTE characteristics; series 2 (black bars) represents operative findings. Scale: 1, none; 2, mild; 3, moderate; 4, severe. Assessment of subvalvular pathology was subjective.

included patient demographic characteristics, preoperative clinical presentations, catheterization data, operative findings, complications of surgery, and outcomes. In addition, in an observational study of 14 consecutive cases of MR, the operative findings were compared with TTE findings. The operative findings noted included site of leaflet tearing, subvalvular pathology (scaled from 1-4 in severity: 1 , none; 2 , mild; 3 , moderate; 4 , severe), and calcification (1, none; 2 , few specks in leaflets; 3 , diffuse involvement in leaflets; 4-paracommissural calcium).

\section{TTE Assessment of Mitral Valves}

The mitral valve of all the patients was assessed by TTE. The assessment included valve mobility and pliability, severity of subvalvular pathology, and leaflet and annular calcium The patients were accordingly classified as high or low risk. ${ }^{4}$ Pliability of the valve leaflets was the primary criterion for selecting patients for the procedure. From 1998, transesophageal echocardiography (TEE) examination was also performed in all cases to exclude left atrial clot.

\section{PTMC Procedure}

All patients had a femoral transvenous antegrade approach. Under fluoroscopy (left anterior oblique view), septal puncture was performed with a Brockenbrough needle. ${ }^{5}$ After septal puncture, mitral valve dilatation was done with an Inoue balloon in stepwise manner. ${ }^{5}$ MR was suspected from elevated left atrial pressures or appearance of giant V wave and was confirmed by TTE. CT was suspected from unstable hemodynamics, and dye leakage confirmed the diagnosis. A pigtail catheter was inserted under fluoroscopic guidance for tamponade release. In patients with severe MR, the clinical profile was evaluated and monitored by hemodynamic variables. In patients with $\mathrm{CT}$ and persistent hemodynamic compromise, the procedure was abandoned for immediate surgical intervention.

\section{Surgical Procedure}

All patients were operated on within 6 hours of attempted dilatation. Acute MR was treated by mitral valve replacement (MVR). 


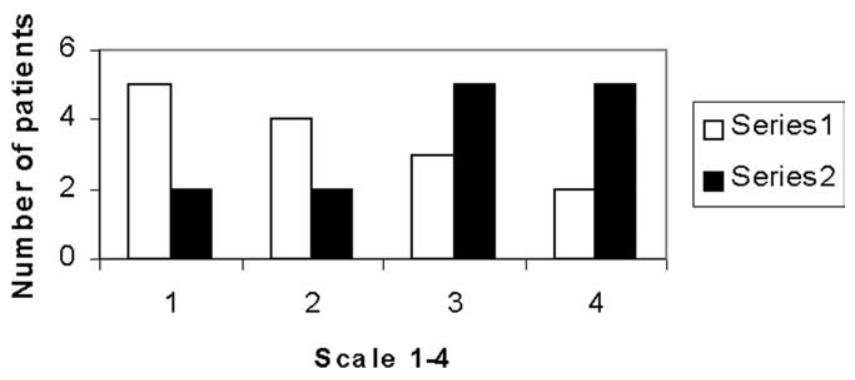

Figure 2. Comparison between TTE findings and operative findings with respect to calcium in 14 consecutive cases of MR. Series 1 (white bars) represents TTE characteristics; series 2 (black bars) represents operative findings. Scale: 1 , none; 2 , few specks in leaflets; 3, diffuse involvement in leaflets; 4, paracommissural calcium.

Septal perforation was repaired directly. CT was managed through a median sternotomy and CPB in 6 cases. One patient with a left atrial appendage (LAA) tear and 1 with a left ventricular tear were operated on through a left anterolateral thoracotomy, and CMV was performed in both patients.

\section{Statistical Analysis}

The statistical analysis was done with SPSS for Windows (version 11.0; SPSS Inc, Chicago, Ill). The continuous variables are expressed as mean $\pm \mathrm{SD}$.

\section{Results}

Of a total of 1388 patients who underwent PTMC in our institution, $31(2.2 \%)$ required emergency surgery. Surgery was for severe MR in 23 patients (74.2\%) and for CT in $8(25.8 \%)$. Mean interval to surgery was $2.8 \pm 2.4$ hours. Patient demographic characteristics are listed in Table 1.

\section{Preoperative Clinical Profile}

The clinical profile is described in Table 2. Three patients with CT had cardiac asystole. In patients with MR, the mean left atrial pressure was $36.1 \pm 12.3 \mathrm{~mm} \mathrm{Hg}$ (range 18-52 $\mathrm{mm} \mathrm{Hg}$ ), and mean $\mathrm{V}$-wave pressure was $61.3 \pm 22.2 \mathrm{~mm}$ $\mathrm{Hg}$ (range 40-92 $\mathrm{mm} \mathrm{Hg}$ ).

\section{Operative Findings}

Of the 23 patients with MR, $10(43.5 \%)$ had central tearing of the anterior mitral leaflet (AML), 10 (43.5\%) had paracommissural leaflet tearing with annular involvement, and 3 (13\%) had central tearing of the posterior mitral leaflet.

CT occurred in 8 cases: before dilatation in 7 patients and during dilatation in 1 patient. The causes of CT included left atrial roof puncture in 4 cases, LAA tearing in 2 cases, left ventricular tearing in 1 case, and right atrial roof tearing in one case.

\section{Correlation Between Echocardiographic Assessment and Surgical Findings}

At surgery, it was found that the TTE scores underestimated the severity of the mitral valve disease. The observed pathology was more severe than that recorded by TTE (Figures 1 and 2), confirming that TTE underestimated the severity of the disease.

\section{Mortality and Complications of Surgery}

MVR was done in 29 cases and CMV in 2 cases. Three patients died after emergency surgery (mortality 9.6\%): 1 patient with left ventricular tearing died on the table because of intractable ventricular fibrillation, and 2 other patients died in the intensive care unit. The patient with left ventricular tearing had cardiac asystole; the tamponade was released by left thoracotomy, and he was resuscitated. During CMV, however, intractable VF developed. One patient, who had juvenile MS and underwent MVR for posterior mitral leaflet tearing, developed gangrene as a result of thrombotic occlusion of right common femoral artery secondary to a retained sheath; the patient had to undergo above knee amputation of the right lower limb and later died of septicemia. The other patient, who had severe pulmonary artery hypertension and right ventricular dysfunction, died of low cardiac output after MVR for AML tearing.

Nine patients (29\%) had postoperative low cardiac output; among them, 1 patient each required intra-aortic balloon counterpulsation and delayed sternal closure. Another patient had persistent coagulopathy, which resolved during the course of 11 days. Mean intensive care unit stay was $4.6 \pm 3.8$ days (range 2-11 days); mean hospital stay was $10.2 \pm 4.6$ days (range 8-16 days).

\section{Discussion}

Development of severe MR is one of the most dreaded complications of PTMC. It may result in pulmonary edema and even death. This complication has been observed in $6 \%$ to $7.5 \%$ of patients who undergo Inoue balloon PTMC. ${ }^{6}$ Various mechanisms - such as tearing of leaflets, rupture of papillary muscles, ${ }^{7}$ rupture of chordae tendineae, ${ }^{8}$ excessive splitting of the commissures, ${ }^{9}$ and mild leaflet prolapse ${ }^{10}$ may account for the development of MR. In our series, only the leaflet tearing caused acute, severe MR necessitating emergency surgery. The leaflet tearing led to severe elevation of left atrial pressure, and in the presence of unrelieved MS, the clinical picture of pulmonary edema ensued. Most of our patients had pulmonary arterial hypertension before PTMC (mean pulmonary artery pressure $68.6 \pm 26.2 \mathrm{~mm}$ $\mathrm{Hg}$ ). Acute MR in the presence of severe pulmonary arterial hypertension leads to hemodynamic compromise. ${ }^{11}$

In our series, 3 patients with acute MR had previous CMV. The mitral valve was distorted after previous commissurotomy. More often, the split commissural area was 


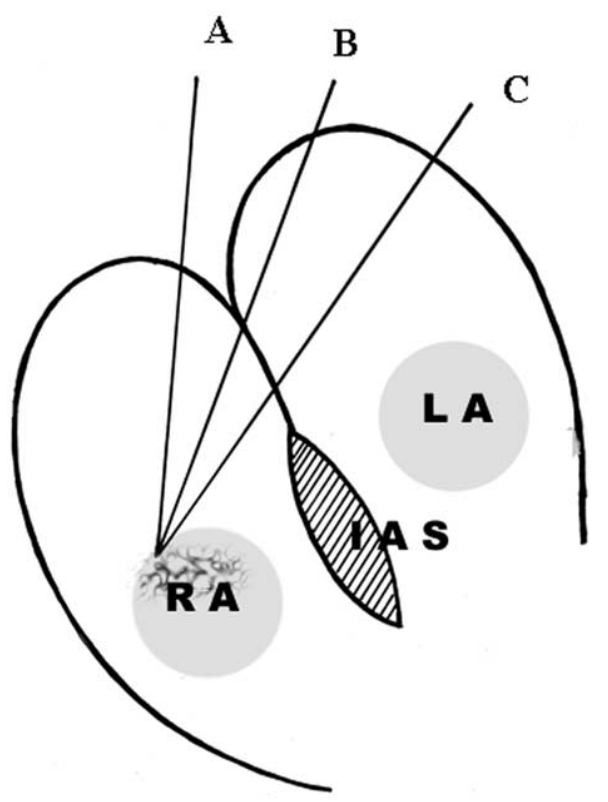

Figure 3. Various mechanisms of CT in our series. In presence of enlarged right atrium (RA) or left atrium (LA), interatrial septum (IAS) is displaced inferiorly, leading to high puncture. $A$ and $B$ lead to roof puncture, whereas $C$ leads to LAA perforation.

heavily fibrosed and thickened, with severe subvalvular pathology. Even though excellent results have been reported for one large series, ${ }^{12}$ the natural progression of rheumatic disease with the fibrocicatricial changes of commissurotomy could make the mitral valve anatomy heavily deformed, leading to transmission of balloon pressure in an unpredictable way that would cause valve damage.

\section{Morphology and Mechanism of Leaflet Tearing}

On the basis of our study of 14 consecutive cases of MR, we propose the following mechanism of leaflet tearing. The balloon delivers sudden pressure to the fused leaflets, which split along the area of least resistance; that is, usually along the commissural fusion. Commissural and paracommissural fibrocalcific dystrophy represent sites of greater resistance that hinder commissural splitting, leading to delivery of the balloon pressure to the relatively thin AML and causing it to tear. The AML was involved in 13 cases, because the posterior mitral leaflet was fibrosed and rolled up. The AML tearing occurred in its central or paracommissural areas in all the cases studied.

\section{Acute MR and Its Treatment}

Most reports describe management of mitral leaflet disruption with MVR. Certain other groups have had success in mitral valve repair with complex repair techniques. ${ }^{13}$ In our series, the valve commissures were often severely fibrotic, with a thickened and fibrotic valve with severe subvalvular pathology and paracommissural calcium, making the valve unsuitable for repair. Leaflet tears were often irregular and extending to and involving the mitral annulus, which was a deterrent for attempts at valve repair.

\section{Mechanism of Atrial Perforation}

The mechanism of atrial perforation in our series is depicted in Figure 3. The interatrial septal puncture is the critical step in performing the PTMC. In the presence of left or right atrial enlargement, the free wall enlarges while the interatrial septum is pushed relatively inferior. Therefore the likelihood of a high septal puncture is enhanced in the presence of an enlarged left or right atrium. In three of the cases of left atrial roof tearing, the left atrium was larger than $50 \mathrm{~mm}$. Two patients had LAA tearing as a result of the needle and sheath perforating the appendage during septal puncture. The 1 patient with right atrial tearing had moderately severe tricuspid regurgitation and an enlarged right atrium. In patients with MS, in whom the normal geometry is lost because of biatrial enlargement, septal puncture should be undertaken with extreme caution. ${ }^{14}$ TEE-guided septal puncture can be helpful in this situation. ${ }^{15}$

\section{Mechanisms of Left Ventricular Tearing}

In 1 patient, the left ventricle was dilated with the Inoue balloon, leading to laceration of the left ventricular wall, CT, and cardiac arrest. The patient had juvenile rheumatism and small left ventricle. Slippage of the balloon to the left ventricle, combined with the left ventricle's small size, caused the disruption. Proper placement of balloon in the mitral valve under echocardiographic guidance might have prevented this complication.

\section{Management of CT}

When hemopericardium was diagnosed with hemodynamic compromise, patients were shifted to the operating room for surgery. Six patients underwent exploration through a median sternotomy with cardiopulmonary bypass. After the tearing was controlled, MVR was done. Open mitral valvotomy was not performed because of poor preoperative hemodynamics. CMV was performed in 2 cases. In 1 patient, the site of tearing was localized to the LAA by preoperative fluoroscopy; we therefore proceeded with CMV. The other patient required stroke thoracotomy for release of tamponade, resuscitation, and control of ventricular tearing. After the tearing was controlled, CMV was performed.

\section{Mortality and Morbidity}

The cost and time constraints of having an operating room and the surgical and anesthesia team on standby have led many centers to relax this practice; however, this has also proved costly at times. ${ }^{3}$ Ours is a large-volume center for 
PTMC, and as many as $20 \%$ of valves dilated fall into the high-risk category. We therefore routinely provide surgical standby for PTMC. There was significant mortality and morbidity in the postoperative period. Sudden elevation of right ventricular systolic pressure as a result of MR and hypotension could lead to right ventricular subendocardial ischemia, which may manifest as low cardiac out put in the postoperative period. Early ventilation and surgery, with the capacity for an on site-circulatory support system, ${ }^{3}$ could decrease adverse outcomes.

\section{Study Limitations}

The major limitation of this study is its retrospective nature, spanning 14 years and involving a limited number of patients. During this period, factors such as change in the technique of PTMC and selection of cases may have influenced the incidence of complications. Until 1998, CMV was the mainstay of management of MS at our institution. With increasing operative experience, however, many high-risk patients have been accepted for PTMC, and by 2000 most of our cases were managed with PTMC. This has increased the incidence of complications, with 25 complications recorded in a 5-year period from 1999 to 2003. TEE was introduced in 1998; however, its use was restricted to ruling out left atrial clots. Valve morphology was primarily assessed by TTE; this significant limitation may explain the discrepancies between operative and echocardiographic findings.

\section{Conclusion}

Emergency surgery after PTMC is rare, with acute, severe MR and CT the conditions that necessitate it. TTE often underestimates the severity of mitral valve disease. Severe commissural pathology, paracommissural calcium, and pliable leaflet were seen with AML tearing. Septal puncture is the critical event in PTMC. Inadvertent high puncture may lead to left or right atrial roof perforation. PTMC in juvenile patients could lead to left ventricular tearing.

We acknowledge the help of Mr. Lijjikumar and Ms Vasanthi in the preparation of figures.

\section{References}

1. Inoue K, Owaki T, Nakamura T, Kitamura F, Miyamoto N. Clinical application of transvenous mitral commissurotomy by a new balloon catheter. J Thorac Cardiovasc Surg. 1984;87:394-402.

2. O'Shea JP, Abascal VM, Wilkins GT, Marshall JE, Brandi S, Acquaella $\mathrm{H}$, et al. Unusual sequelae after percutaneous mitral valvuloplasty: a Doppler echocardigraphic study. J Am Coll Cardiol. 1992; 19:186-91.

3. Acar C, Deloche A, Tibi PR, Jabara V, Chachques JC, Fabiani JN, et al. Operative findings after percutaneous mitral dilation. Ann Thorac Surg. 1990;49:959-63.

4. Wilkins GT, Weyman AE, Abascal VM, Block PC, Palacios IF. Percutaneous mitral valvotomy: an analysis of echocardiographic variables related to outcome and the mechanism of dilation. Br Heart J. 1988;60:299-308.

5. Vahanian A, Iung B, Cormier B. Mitral valvuloplasty. In: Topol EL, editor. Textbook of interventional cardiology. 4th ed. Philadelphia: WB Saunders; 2003. p. 921.

6. Complications and mortality of percutaneous balloon mitral commissurotomy. A report from the National Heart, Lung, and Blood Institute Balloon Valvuloplasty Registry. Circulation. 1992;85:2014-24.

7. Acar C, Vahanian A, Deloche A, Acar J, Carpentier A. Traumatic rupture of papillary muscle after percutaneous commissurotomy. J Thorac Cardiovasc Surg. 1990;99:376-8.

8. Chern MS, Chang HJ, Lin FC, Wu D. String-plucking as a mechanism of chordal rupture during balloon mitral valvuloplasty using Inoue balloon catheter. Catheter Cardiovasc Interv. 1999;47:213-7.

9. Kaul UA, Singh S, Kalra GS, Nair M, Mohan JC, Nigam M, et al. Mitral regurgitation following percutaneous transvenous mitral commissurotomy. J Heart Valve Dis. 2000;9:262-6.

10. Essop MR, Wisenbaugh T, Skoularigis J, Middlemost S, Sareli P. Mitral regurgitation following mitral balloon valvotomy. Differing mechanisms for severe versus mild-to-moderate lesions. J Heart Valve Dis. 1991;84:1669-79.

11. Tempe DK, Mehta N, Mohan JC, Tandon MS, Nigam M. Early hemodynamic changes following emergency mitral valve replacement for traumatic mitral insufficiency following balloon mitral valvotomy: Report of six cases. Anesthesiology 1998;89:1583-85.

12. Sharma S, Loya YS, Desai DM, Pinto RJ. Balloon valvotomy for mitral restenosis after open or closed surgical commissurotomy. Int J Cardiol. 1993;39:103-8.

13. Acar C, Vahanian A, Jebara VA, Fabiani JN, Deloche A, Acar J, et al. Mitral valve repair for anterior leaflet disruption after percutaneous dilation. J Thorac Cardiovasc Surg. 1991;102:468-9.

14. Holmes DR Jr, Hildner FJ. Transseptal catheterization 1992: it is here to stay. Cathet Cardiovasc Diagn. 1992;26:264-5.

15. Ballal RS, Mahan EF, Nanda NC, Dean LS. Utility of transesophageal echocardiography monitoring in interatrial septal puncture during percutaneous mitral balloon commissurotomy. Am J Cardiol. 1990;66: $230-2$. 